Pacific

Journal of

Mathematics

EARTHQUAKES AND THURSTON'S BOUNDARY

FOR THE TEICHMÜLLER SPACE

OF THE UNIVERSAL HYPERBOLIC SOLENOID

DRAGOMIR ŠARIĆ 


\title{
EARTHQUAKES AND THURSTON'S BOUNDARY FOR THE TEICHMÜLLER SPACE OF THE UNIVERSAL HYPERBOLIC SOLENOID
}

\author{
DRAGOMIR ŠSARIĆ
}

\begin{abstract}
A measured lamination on the universal hyperbolic solenoid $\mathscr{S}$ is, by our definition, a leafwise measured lamination with appropriate continuity for the transverse variations. An earthquakes on the universal hyperbolic solenoid $\mathscr{S}$ is uniquely determined by a measured lamination on $\mathscr{Y}$; it is a leafwise earthquake with the leafwise earthquake measure equal to the leafwise measured lamination. Leafwise earthquakes fit together to produce a new hyperbolic metric on $\mathscr{S}$ which is transversely continuous, and we show that any two hyperbolic metrics on $\mathscr{S}$ are connected by an earthquake. We also establish the space of projective measured lamination $\operatorname{PML}(\mathscr{S})$ as a natural Thurston-type boundary to the Teichmüller space $T(\mathscr{S})$ of the universal hyperbolic solenoid $\mathscr{T}$. The baseleaf-preserving mapping class group $M C G_{B L P}(\mathscr{S})$ acts continuously on the closure $T(\mathscr{S}) \cup P M L(\mathscr{P})$ of $T(\mathscr{Y})$. Moreover, the set of transversely locally constant measured laminations on $\mathscr{S}$ is dense in $\operatorname{ML}(\mathscr{S})$.
\end{abstract}

\section{Introduction}

The universal hyperbolic solenoid $\mathscr{Y}$ is the inverse limit of the system of all finite sheeted unbranched pointed covers of a compact surface of genus greater than one. Sullivan [1993] introduced the solenoid $\mathscr{Y}$ as the universal compact surface, that is, the universal object in the category of finite unbranched covers of a compact surface. $\mathscr{S}$ has a rich deformation theory; its Teichmüller space $T(\mathscr{Y})$ is complex being in fact the first example of a Teichmüller space which is separable but not finite dimensional. Recall that Teichmüller spaces of compact Riemann surfaces with finite points possibly removed are finite-dimensional complex manifolds, and that Teichmüller spaces of geometrically infinite Riemann surfaces are nonseparable infinite-dimensional complex Banach manifolds.

MSC2000: primary 30F60, 30F45, 32H02, 32G05; secondary 30C62.

Keywords: earthquakes, solenoid, laminations, Thurston's boundary. 
Sullivan started in the same paper the study of the complex structure and Teichmüller metric on $T(\mathscr{S})$. Unlike most laminations, the universal hyperbolic solenoid $\mathscr{S}$ has a transverse measure, which is used in [Šarić 2004b] to continue the investigation of Teichmüller metric on $T(\mathscr{Y})$. The results in that paper are used in [Epstein et al. 2006] to show that generic points in $T(\mathscr{S})$ do not have Teichmüllertype Beltrami coefficient representatives, which sharply contrasts the situations for finite surfaces, where all points have Teichmüller type representatives, and for infinite surfaces, where a dense, open subset of the Teichmüller space does. Thus, unlike Riemann surfaces, most points in $T(\mathscr{S})$ are not connected to the basepoint by a nice Teichmüller geodesic obtained by stretching horizontal and shrinking vertical foliations of a holomorphic quadratic differential on $\mathscr{S}$ (see [Lakic 1997] for the statement for infinite surfaces).

According to the results of [Candel 1993], a conformal structure on $\mathscr{Y}$ contains a unique hyperbolic metric. The present paper starts the investigation of Teichmüller space $T(\mathscr{S})$ of the solenoid $\mathscr{Y}$ using the hyperbolic structures on $\mathscr{Y}$. The approach to study the decorated Teichmüller space of the related punctured solenoid via hyperbolic structures is made in [Penner and Šarić 2005; Bonnot et al. 2006]. That approach is specific to the punctured solenoid while the results in this paper hold for both universal hyperbolic solenoid and punctured solenoid.

Our first result concerns earthquakes between two hyperbolic solenoids. We recall the notion of an earthquake; see [Thurston 1986] for details. An earthquake on a hyperbolic surface is a piecewise isometric bijective map from the hyperbolic surface to another hyperbolic surface, which is not necessarily continuous. The support of an earthquake is a geodesic lamination, called the earthquake fault, along which the quaking, that is the discontinuity, appears. The restriction of an earthquake to each stratum that is a leaf of the support or a connected component of the complement of the support is an isometry, and each stratum is moved to the left when viewed from any other stratum. Given an earthquake on a hyperbolic surface, there exists a unique transverse measure, called an earthquake measure, to its support which determines the earthquake. An earthquake measure is identified with a positive Radon measure, called a measured lamination, on the space of geodesics of a surface whose support is the support geodesic lamination; the measure of a bunch of geodesics in the support is given by the measure of an arc which intersects them and which does not intersect other geodesics in the support. The main result concerning the earthquakes on hyperbolic surfaces is that any two hyperbolic metrics are connected by an earthquake.

We introduce a proper notion of an earthquake measure on the universal hyperbolic solenoid $\mathscr{Y}$. An earthquake measure on $\mathscr{Y}$ is an assignment of an earthquake measure on each leaf, isometric to a hyperbolic plane, of $\mathscr{S}$ such that the measures vary continuously for the transverse variations in an appropriate topology 
(see Definition 4.2 for more details). The support of leafwise earthquake measures are leafwise geodesic laminations which do not vary continuously for the transverse variations (see Example 4.3). However, the continuity of measures in the appropriate Fréchet topology guarantees that the leafwise earthquakes on $\mathscr{S}$ piece together a new hyperbolic structure on $\mathscr{Y}$, which is continuous for the transverse variations. Therefore, earthquake measures on $\mathscr{S}$ produce an earthquake of $\mathscr{Y}$. We establish the transitivity statement for earthquakes on hyperbolic structures of the universal hyperbolic solenoid $\mathscr{Y}$, analogous to the case of hyperbolic surfaces.

Theorem 5.1. A measured lamination $\mu$ on a solenoid $X$ with an arbitrary hyperbolic metric gives an earthquake map $E_{\mu}$ of $X$ into another solenoid $Y$ with a hyperbolic metric such that there exists a (differentiable) quasiconformal map $f: X \rightarrow Y$ whose extension to the boundary of leaves coincides with the extension of $E_{\mu}$. Any two points in the Teichmüller space $T(\mathscr{Y})$ of the universal hyperbolic solenoid $\mathscr{S}$ are connected by a unique earthquake along a measured lamination.

To prove this, we show that if two quasisymmetric maps of the unit circle $S^{1}$ are close then their corresponding earthquake measures are close in the Fréchet topology. The opposite is false by an easy example. Therefore, the earthquake map from the space of bounded measured laminations in the unit disk onto the Teichmüller space is bijective but not a homeomorphism for the Fréchet topology on measured lamination. In the case of compact surfaces, the earthquake map is a homeomorphism if measured laminations are equipped with the weak-* topology, which is equivalent to the Fréchet topology for compact surfaces [Kerckhoff 1983]. We establish similar result for the solenoid $\mathscr{Y}$.

Corollary 5.2. The earthquake map that assigns to each bounded measured lamination on the universal hyperbolic solenoid $\mathscr{Y}$ the corresponding marked hyperbolic solenoid is a homeomorphism between the space $M L(\mathscr{Y})$ of bounded measured laminations and the Teichmüller space $T(\mathscr{Y})$.

Thurston [1988] (see also [Fathi et al. 1979]) introduced a natural boundary for the Teichmüller space of a compact surface by adding at infinity to the space of projective measured laminations. The mapping class group acts continuously on the closure and there is a classification of its elements according to their actions on the boundary. Bonahon [1988] gave an alternative description of Thurston's boundary to the Teichmüller space of a compact surface using the Liouville map which embeds the Teichmüller space into the space of measures on the space of geodesics of the surface. The boundary points at infinity are asymptotic rays to the image of the Teichmüller space. In [Šarić 2005] we used the idea of the Liouville embedding to give a Thurston-type boundary to the Teichmüller space of any Riemann surface, possibly geometrically infinite. We extend this result to the Teichmüller space $T(\mathscr{S})$ of the universal hyperbolic solenoid $\mathscr{Y}$. 
Biswas, Nag and Mitra introduced in [Biswas et al. 1999] the direct limit of projective measured laminations on the compact surfaces as boundary at infinity of the direct limit of Teichmüller spaces of compact surfaces covering a fixed compact surface of genus at least two. Since $T(\mathscr{S})$ contains the above direct limit of Teichmüller spaces of compact surfaces as a dense subset, the authors remark that Thurston's boundary for $T(\mathscr{Y})$ should be a completion of the union of the projective measured laminations on all compact surfaces. We give a proper analytical description of the Thurston's boundary that answers their question about the completion. The main point is to properly define continuity for the transverse variations of various spaces of measures and distributions on the space $\mathscr{G}(\mathscr{S})$ of geodesics on the universal hyperbolic solenoid $\mathscr{Y}$. We establish this goal using the Fréchet topology on the enveloping space of Hölder distributions $H(\mathscr{S})$ in Section 6.

Theorem 6.2. The Liouville map $\mathscr{L}_{\mathscr{S}}: T(\mathscr{Y}) \rightarrow H(\mathscr{Y})$ is a homeomorphism onto its image. The set of asymptotic rays to $\mathscr{L}_{\mathscr{S}}(T(\mathscr{S}))$ is homeomorphic to $\operatorname{PML}(\mathscr{S})$, the space of projective measured laminations on $\mathscr{S}$. The baseleaf-preserving mapping class group $M C G_{B L P}(\mathscr{S})$ acts continuously on the closure $T(\mathscr{Y}) \cup P M L(\mathscr{S})$ of the Teichmüller space $T(\mathscr{Y})$ of the universal hyperbolic solenoid $\mathscr{S}$.

A lift of a measured lamination on a compact surface of genus at least two to the universal hyperbolic solenoid $\mathscr{Y}$ is a measured lamination on $\mathscr{Y}$. Such a measured lamination is locally constant for transverse variations and is called a transversely locally constant (TLC) measured lamination. We show that an arbitrary measured lamination on $\mathscr{S}$ is the limit of TLC measured laminations in the Fréchet topology.

Theorem 6.3. The subset of all measured laminations on the universal hyperbolic solenoid 9 that are locally transversely constant, is dense in the space of all measured laminations $M L(\mathscr{Y})$ on $\mathscr{Y}$ for the Fréchet topology.

In Section 7, we introduce the space of compactly supported measured laminations $P M L_{0}\left(\mathscr{Y}_{p}\right)$ on the punctured solenoid $\mathscr{Y}_{p}$ and extend the transitivity of earthquakes and Thurston's boundary for the punctured solenoid by replacing $M L(\mathscr{Y})$ with $M L_{0}\left(\mathscr{S}_{p}\right)$.

\section{Preliminaries}

We recall several definitions: the universal hyperbolic solenoid, earthquakes of the unit disk, the Fréchet topology on the measures on the space of geodesics of the unit disk.

The universal hyperbolic solenoid. Let $(S, x)$ be a fixed compact surface of genus at least two with the basepoint $x \in S$. Consider all finite sheeted unbranched covers $\left(S_{i}, x_{i}\right)$ by compact surfaces with basepoints such that the covering maps $\pi_{i}: S_{i} \rightarrow S$ satisfy $\pi_{i}\left(x_{i}\right)=x$. There is a natural partial ordering $\leq$ on the set 
of all such coverings. Namely, $\left(S_{i}, x_{i}\right) \leq\left(S_{j}, x_{j}\right)$ if there exists a finite sheeted unbranched covering map $\pi_{j, i}: S_{j} \rightarrow S_{i}$ such that $\pi_{j, i}\left(x_{j}\right)=x_{i}, \pi_{i} \circ \pi_{j, i}=\pi_{j}$. The set of all coverings is inverse directed, that is, given two coverings of $S$, there exists a third covering of $S$ which is larger than those two; see [Sullivan 1993; Šarić 2004b; Odden 2005; Markovic and Šarić 2006].

Definition 2.1 [Sullivan 1993]. The universal hyperbolic solenoid $\mathscr{Y}$ is the inverse limit of the directed system of all finite sheeted unbranched covers of a fixed compact surface of genus at least two for the above partial ordering.

The inverse limit $\mathscr{Y}$ is independent of the base surface, that is two inverse limits with two base surfaces of genus at least two are homeomorphic. Thus it is called the universal hyperbolic solenoid $\mathscr{Y}$.

We give an equivalent definition of the universal hyperbolic solenoid $\mathscr{S}$; the reference for most of this paragraph is [Odden 2005]. Let $G$ be a Fuchsian group uniformizing $S$, that is $S$ is homeomorphic to $\boldsymbol{D} / G$ where $\boldsymbol{D}$ is the unit disk. Let $G_{n}$ be the intersection of all subgroups of $G$ with index at most $n$. Then $G_{n}$ is of finite index in $G$. We define a profinite metric on $G$ by

$$
\operatorname{dist}(A, B)=e^{-n},
$$

for $A, B \in G$, where $A B^{-1} \in G_{n}$ and $A B^{-1} \notin G_{n+1}$. The completion of $G$ in the profinite metric dist is called the profinite group completion $\hat{G}$. The completion $\hat{G}$ is a compact topological group homeomorphic to Cantor set. The group $G$ is a dense subgroup of $\hat{G}$. We define an action of $G$ on the product $\boldsymbol{D} \times \hat{G}$ by

$$
A(z, t):=\left(A(z), t A^{-1}\right),
$$

where $z \in \boldsymbol{D}$ and $t \in \hat{G}$, the action of $A$ on $\boldsymbol{D}$ is just a Möbius map, and $A$ acts on $\hat{G}$ because $G$ lies inside $\hat{G}$. The universal hyperbolic solenoid $\mathscr{S}$ is homeomorphic to the quotient $(\boldsymbol{D} \times \hat{G}) / G$. From this description of $\mathscr{Y}$, it is easy to see that $\mathscr{S}$ is a compact space which is locally homeomorphic to a 2-disk times Cantor set. Moreover, $\mathscr{S}$ fibers over $\boldsymbol{D} / G$ with fibers Cantor sets isomorphic to $\hat{G}$, and the restriction of the fiber map to each leaf is the universal covering of $S \equiv \boldsymbol{D} / G$. The same is true for any finite cover of $S$ by replacing $G$ with the covering group [Odden 1997]. Each $(\boldsymbol{D} \times\{t\}) / G \subset \mathscr{Y}$, for $t \in \hat{G}$, is a path component, called a leaf of $\mathscr{Y}$. Each leaf is homeomorphic to the unit disk and it is dense in $\mathscr{Y}$. Thus each leaf is simply connected but the restriction of the topology on $\mathscr{Y}$ to a leaf is not the standard topology on the unit disk. We define the universal cover of $\mathscr{Y}$ by straightening the topology on leaves.

Definition 2.2. The universal cover for the universal hyperbolic solenoid $\mathscr{Y}$ is given by $\pi: D \times \hat{G} \rightarrow(\boldsymbol{D} \times \hat{G}) / G$, where $\pi$ is the quotient map for the action of $G$. 
A complex structure on the universal hyperbolic solenoid $\mathscr{Y}$ is a collection of local charts such that the transition maps are holomorphic in the disk direction and they vary continuously for topology of uniform convergence in the transverse Cantor direction of the local charts [Sullivan 1993]. Complex structures on $\mathscr{Y}$ are in one to one correspondence with conformal structures on $\mathscr{Y}$ that are continuous for the variations in the transverse direction, via the continuous dependence on the parameters of the solution of Beltrami equation. Any conformal structure on $\mathscr{Y}$ contains a unique hyperbolic metric which is continuous for the variations in the transverse direction [Candel 1993]. A hyperbolic metric (or a complex structure) on $\mathscr{Y}$, which is transversely locally constant for some choice of charts on $\mathscr{Y}$, is called a TLC hyperbolic metric (or a TLC complex structure) on $\mathscr{S}$ [Sullivan 1993]. Any TLC hyperbolic metric (or TLC complex structure) on $\mathscr{Y}$ is obtained by lifting a hyperbolic metric (or a complex structure) from a finite cover of $S$ to $\mathscr{Y}$ [Nag and Sullivan 1995]. Note that we fix a TLC complex structure on $\mathscr{S}$ coming from Riemann surface $\boldsymbol{D} / G$ by identifying $\mathscr{S}$ with $(\boldsymbol{D} \times \hat{G}) / G$. A (differentiable) quasiconformal map $f: \mathscr{Y} \rightarrow X$ from the fixed TLC complex solenoid $\mathscr{Y}$ to an arbitrary complex solenoid $X$ is a homeomorphism which is $C^{\infty}$-differentiable in the disk direction in local charts and varies continuously in the transverse direction for $C^{\infty}$-topology on $C^{\infty}$-maps [Sullivan 1993; Šaric 2004b]. We note that the use of differentiable quasiconformal maps as opposed to only quasiconformal maps is necessary in order for the compositions of quasiconformal maps to be continuous in the transverse direction. We define the Teichmüller space $T(\mathscr{S})$ of the universal hyperbolic solenoid $\mathscr{Y}$ as follows.

Definition 2.3. The Teichmüller space $T(\mathscr{S})$ of the universal hyperbolic solenoid $\mathscr{Y}$ consists of all quasiconformal maps $f: \mathscr{S} \rightarrow X$ up to an equivalence. Two quasiconformal maps $f: \mathscr{Y} \rightarrow X$ and $g: \mathscr{S} \rightarrow X_{1}$ are equivalent if there exists a conformal map $c: X \rightarrow X_{1}$ such that $g^{-1} \circ c \circ f: \mathscr{Y} \rightarrow \mathscr{Y}$ is homotopic to the identity. The equivalence class of the identity id : $\mathscr{S} \rightarrow \mathscr{S}$ is called the basepoint of $T(\mathscr{\varphi})$.

Earthquakes in the unit disk. We define earthquakes of the unit disk $\boldsymbol{D}$ and recall their main properties. A geodesic lamination in the unit disk $\boldsymbol{D}$ is a closed subset of $\boldsymbol{D}$ which is foliated by geodesics for the hyperbolic metric on $\boldsymbol{D}$, or equivalently, it is a closed subset of the space $\mathscr{G}(\boldsymbol{D})$ of geodesics in $\boldsymbol{D}$ such that no two geodesics in the subset intersect in $\boldsymbol{D}$. Some familiar examples of geodesic laminations in $\boldsymbol{D}$ are: a set of finitely many nonintersecting geodesics in $\boldsymbol{D}$; a countable, discrete set of nonintersecting geodesics; a foliation of $\boldsymbol{D}$ by geodesics.

Definition 2.4. An earthquake measure on the unit disk is a positive Radon measure on the space of geodesic $\mathscr{G}(\boldsymbol{D})$ of the unit disk $\boldsymbol{D}$, whose support is a geodesic lamination. 
Note that $\mathscr{G}(\boldsymbol{D})$ is homeomorphic to $\left(S^{1} \times S^{1}-\operatorname{diag}\right) / \mathbb{Z}_{2}$ by mapping a geodesic in $\boldsymbol{D}$ to the unordered pair of its ideal endpoints in $S^{1}$. Earthquakes were introduced in [Thurston 1986] as follows (this is the reference for all results on this page unless otherwise specified).

Definition 2.5. An earthquake $E: D \rightarrow D$ of the unit disk $\boldsymbol{D}$ is a bijective map which maps a fixed geodesic lamination $\lambda$, called the support of $E$, in $\boldsymbol{D}$ onto another geodesic lamination $\lambda^{\prime}$. A geodesic from $\lambda$ or a connected component of $D-\lambda$ is called a stratum of $E$. The restriction of the earthquake $E$ to each stratum is a hyperbolic isometry with the additional property that for any two strata $A, B$ of $E$, the comparison isometry $\left.E\right|_{B} \circ\left(\left.E\right|_{A}\right)^{-1}$ is a hyperbolic translation whose axis separates $A$ from $B$, and which translates $B$ to the left as seen from $A$.

Each earthquake $E: \boldsymbol{D} \rightarrow \boldsymbol{D}$ continuously extends to a homeomorphism of $\partial \boldsymbol{D} \equiv S^{1}$, which we denote by $\left.E\right|_{S^{1}}: S^{1} \rightarrow S^{1}$. An important theorem due to Thurston is that each orientation-preserving homeomorphism of $S^{1}$ is obtained as the extension of an earthquake to $S^{1}$. Given an earthquake $E$ of $\boldsymbol{D}$, there exists a unique corresponding earthquake measure $\mu$ on $\mathscr{G}(\boldsymbol{D})$ supported on $\lambda$ determined by the following condition. Consider a subset of $\lambda$ consisting of geodesics which intersect a closed $\operatorname{arc} I$. Choose finitely many strata of $E$ intersecting $I$. The $\mu$ measure of the subset is approximated by the sum of translation lengths between comparison isometries of adjacent strata of the above chosen finitely many strata of $E$ intersecting the arc $I$, when the distance between adjacent strata goes to zero. An alternative description is to consider the measure $\mu$ to be a family of measures on $\operatorname{arcs} I$ in $\boldsymbol{D}$ which are invariant under homotopies of arcs preserving the leaves of $\lambda$. If an earthquake measure $\mu$ corresponds to an earthquake as above, we denote the corresponding earthquake by $E_{\mu}$. Two homeomorphisms have the same corresponding earthquake measures if and only if they differ by a postcomposition with a hyperbolic isometry of $\boldsymbol{D}$.

We say that an earthquake measure $\mu$ is bounded if the norm $\|\mu\|$ satisfies

$$
\|\mu\|:=\sup _{I}\{\mu(I)\}<\infty,
$$

where the supremum is over all geodesic $\operatorname{arcs}$ in $\boldsymbol{D}$ of length 1 . If $\mu$ is a bounded earthquake measure, then there exists earthquake $E_{\mu}$ corresponding to $\mu$. A homeomorphism $h: S^{1} \rightarrow S^{1}$ is quasisymmetric if and only if the corresponding earthquake measure $\mu$ is bounded, where $\left.E_{\mu}\right|_{S^{1}}=h$ [Šarić 2006; 2007].

The Fréchet topology. We recall the definition of the Fréchet topology on the space of Hölder distributions $H(\boldsymbol{D})$ of the unit disk $\boldsymbol{D}$ from [Šarić 2004a]. The space of bounded positive measures on $\mathscr{G}(\boldsymbol{D})$, and the space of bounded earthquake measures on $\mathscr{G}(\boldsymbol{D})$, are subsets of $H(\boldsymbol{D})$. 
The Liouvile measure $L$ on $\mathscr{G}(\boldsymbol{D})$ is given by

$$
L(K):=\iint_{K} \frac{d \alpha d \beta}{\left|e^{i \alpha}-e^{i \beta}\right|^{2}},
$$

where $e^{i \alpha}, e^{i \beta} \in S^{1}$. A box of geodesics $Q$ is the set of all geodesics in

$$
(a, b) \times(c, d) \subset S^{1} \times S^{1}-\operatorname{diag},
$$

where $a, b, c, d \in S^{1}$ are distinct points in counterclockwise order. Then

$$
L(Q)=\log \left|\frac{(a-c)(b-d)}{(a-d)(b-c)}\right|,
$$

and this formula can be used as an alternative definition of Liouville measure.

We fix $0<v \leq 1$. The space of $v$-test functions, test $(v)$, consists of all $v$-Hölder continuous functions $(\varphi, Q)$ with $\varphi: \mathscr{G}(\boldsymbol{D}) \rightarrow \mathbb{R}$ whose support is in a box of geodesics $Q$ with $L(Q)=\log 2$ such that

$$
\left\|\varphi \circ \Theta_{Q}\right\|_{v} \leq 1
$$

where $\Theta_{Q}:(-1,-i) \times(1, i) \mapsto Q$ is a hyperbolic isometry and

$$
\|\varphi\|_{\nu}:=\max \left\{\sup _{\varphi_{(\boldsymbol{D})}}\{|\varphi|\}, \sup \left\{\frac{\left|\varphi(x, y)-\varphi\left(x_{1}, y_{1}\right)\right|}{d\left((x, y),\left(x_{1}, y_{1}\right)\right)^{v}}:(x, y) \neq\left(x_{1}, y_{1}\right)\right\}\right\},
$$

with $d$ being the angle metric on $S^{1} \times S^{1}$ [Šarić 2004a; 2005].

The space of Hölder distribution $H(\boldsymbol{D})$ of the unit diks $\boldsymbol{D}$ consists of all linear functionals $\Psi$ on the space of Hölder continuous functions $\varphi: \mathscr{G}(\boldsymbol{D}) \rightarrow \mathbb{R}$ with compact support such that

$$
\|\Psi\|_{v}:=\sup \{|\Psi(\varphi)|: \varphi \in \operatorname{test}(\nu)\}<\infty
$$

for all $0<v \leq 1$ [Šarić 2005]. The Fréchet topology on $H(\boldsymbol{D})$ is defined using the family of $v$-norms above. The topological vector space $H(\boldsymbol{D})$ is metrizable and one metric which gives the Fréchet topology is

$$
\operatorname{dist}\left(\Psi, \Psi_{1}\right):=\sum_{n=1}^{\infty} \frac{1}{n^{2}}\left\|\Psi-\Psi_{1}\right\|_{1 / n} .
$$

\section{The convergence of measures in the unit disk}

Denote the set of geodesics in $\boldsymbol{D}$ which contain $z \in \mathscr{G}_{\text {by }} \mathscr{G}_{z}$. If $z, w \in \boldsymbol{D}$ then denote by $[z, w]$ the geodesic arc in $\boldsymbol{D}$ between $z$ and $w$. If $K$ is a subset of $\boldsymbol{D}$, denote by $\varphi_{K}$ the set of geodesics of $\boldsymbol{D}$ which intersect $K$.

We showed in [Šarić 2007] that a sequence of homeomorphisms of $S^{1}$ pointwise converges to a homeomorphism of $S^{1}$ if and only if the sequence of earthquake 
measures, corresponding to the sequence of homeomorphisms, converges to the earthquake measure of the limit. More precisely:

Proposition 3.1 [Šarić 2007]. Let $\mu, \mu_{i}$ be uniformly bounded earthquake measures on $\boldsymbol{D}$, that is $\|\mu\|,\left\|\mu_{i}\right\| \leq M<\infty$. Then $\mu_{i} \rightarrow \mu$ in the weak-* topology as $i \rightarrow \infty$ if and only if there exist normalizations of earthquake maps $\left.E_{\mu_{i}}\right|_{S^{1}},\left.E_{\mu}\right|_{S^{1}}$ such that $\left.\left.E_{\mu_{i}}\right|_{S^{1}}(x) \rightarrow E_{\mu}\right|_{S^{1}}(x)$ for each $x \in S^{1}$, as $i \rightarrow \infty$.

Note that $\left.E_{\mu_{i}}\right|_{S^{1}},\left.E_{\mu}\right|_{S^{1}}$ are well defined up to the postcompositions by isometries and different normalizations correspond to different choices of isometries.

We consider a sequence of quasisymmetric maps converging to a quasisymmetric map in the quasisymmetric topology and show that the corresponding sequence of earthquake measures converges in the Fréchet topology.

Proposition 3.2. Let $h_{n}=\left.E_{\mu_{n}}\right|_{S^{1}}$ and $h=\left.E_{\mu}\right|_{S^{1}}$ be quasisymmetric maps such that $h_{n} \rightarrow h$ as $n \rightarrow \infty$, in the quasisymmetric topology. Then $\left\|\mu_{n}-\mu\right\|_{v} \rightarrow 0$ as $n \rightarrow \infty$, for each $0<v \leq 1$.

Proof. Assume on the contrary that

$$
\left\|\mu_{n}-\mu\right\|_{\nu} \geq m>0
$$

for a fixed $0<v \leq 1$. Then there exists $\left(\varphi_{n}, Q_{n}\right) \in \operatorname{test}(v)$ such that

$$
\left|\mu_{n}\left(\varphi_{n}\right)-\mu(\varphi)\right| \geq m>0,
$$

where $L\left(Q_{n}\right)=\log 2$. Without loss of generality, we assume that $h_{n}, h$ fix $1, i,-1$. Let $Q_{n}^{\prime}:=h_{n}\left(Q_{n}\right)$ and $Q_{n}^{\prime \prime}:=h\left(Q_{n}\right)$. There exist unique hyperbolic isometries $A_{n}, A_{n}^{\prime}, A_{n}^{\prime \prime}$ of the unit disk $\boldsymbol{D}$ such that

$$
\begin{gathered}
A_{n}: Q_{n} \mapsto(1, i) \times(-1,-i), \quad A_{n}^{\prime}: Q_{n}^{\prime} \mapsto(1, i) \times\left(-1, q_{n}^{\prime}\right), \\
A_{n}^{\prime \prime}: Q_{n}^{\prime \prime} \mapsto(1, i) \times\left(-1, q_{n}^{\prime \prime}\right),
\end{gathered}
$$

for unique $q_{n}^{\prime}, q_{n}^{\prime \prime} \in S^{1}$.

Define $\bar{h}_{n}:=A_{n}^{\prime} \circ h_{n} \circ A_{n}^{-1}$ and $\bar{f}_{n}:=A_{n}^{\prime \prime} \circ h \circ A_{n}^{-1}$. Note that

$$
\bar{h}_{n}: 1, i,-1,-i \mapsto 1, i,-1, q_{n}^{\prime}, \quad \bar{f}_{n}: 1, i,-1,-i \mapsto 1, i,-1, q_{n}^{\prime \prime} .
$$

The normalization of $\bar{h}_{n}$ and $\bar{f}_{n}$ implies that $\bar{h}_{n} \rightarrow \bar{h}$ and $\bar{f}_{n} \rightarrow \bar{f}$ pointwise on $S^{1}$, where $\bar{h}, \bar{f}$ are quasisymmetric maps as well. This convergence is a consequence of pointwise convergence of a family of $K$-quasiconformal maps normalized to fix three points in $\hat{\mathbb{C}}$. Note that we can choose quasiconformal extensions of $\bar{h}_{n}, \bar{f}_{n}$ to have the same quasiconformal constant by using barycentric extension in the interior and the exterior of the unit circle $S^{1}$ [Douady and Earle 1986].

Consequently, $\bar{h}_{n} \circ \bar{f}_{n}^{-1} \rightarrow \bar{h} \circ \bar{f}^{-1}$ pointwise, as $n \rightarrow \infty$. Let $Q$ be an arbitrary box with $L(Q)=\log 2$. Then

$$
\left|L\left(A_{n}^{\prime} \circ h_{n} \circ h^{-1} \circ\left(A_{n}^{\prime \prime}\right)^{-1}(Q)\right)-L(Q)\right| \rightarrow 0
$$


as $n \rightarrow \infty$ because $h_{n} \rightarrow h$ in the quasisymmetric topology and by the invariance of Liouville measure under hyperbolic isometries. Thus $\bar{h} \circ \bar{f}^{-1}$ preserves Liouville measure and fixes $1, i,-1$. Therefore, $\bar{h}=\bar{f}$.

Let $\mu_{n}^{\prime}:=A_{n}^{*}\left(\mu_{n}\right)$ and $\sigma_{n}:=A_{n}^{*}(\mu)$. Then there exists a sequence

$$
\left(\varphi_{n}^{\prime},(1, i) \times(-1,-i)\right) \in \operatorname{test}(v)
$$

such that

$$
\left|\mu_{n}^{\prime}\left(\varphi_{n}^{\prime}\right)-\sigma_{n}^{\prime}\left(\varphi_{n}^{\prime}\right)\right| \geq m>0 .
$$

Since $\mu_{n}^{\prime}$ is the pushforward by a hyperbolic isometry of $\mu_{n},\left\|\mu_{n}^{\prime}\right\|=\left\|\mu_{n}\right\|$. Moreover, since $\mu_{n}$ are earthquake measures for $h_{n}$ and $h_{n}$ converges in the quasisymmetric topology, it follows that $\mu_{n}$ are uniformly bounded measures, and that the same holds for $\mu_{n}^{\prime}$. The sequence $\sigma_{n}$ is also uniformly bounded because it is the pushforward of a single measure by hyperbolic isometries. Both sequences $\mu_{n}^{\prime}$ and $\sigma_{n}$ converge to bounded earthquake measures $\mu^{\prime}$ and $\sigma$ such that $\bar{h}=\left.E_{\mu^{\prime}}\right|_{S^{1}}$ and $\bar{f}=\left.E_{\sigma}\right|_{S^{1}}$ by Proposition 3.1. By (1), we conclude that $\mu^{\prime} \neq \sigma$. In fact, the weak-* convergence on a fixed box $(1, i) \times(-1,-i)$ is equivalent to the uniform convergence with respect to all $(\varphi,(1, i) \times(-1,-i)) \in \operatorname{test}(v)$ [Šarić 2004a]. But this is a contradiction with $\bar{h}=\bar{f}$ by the uniqueness of earthquake measures [Thurston 1986].

We remark that the inverse of Proposition 3.2 is not true. This is easily seen by an example. Take a fixed geodesic with a positive weight as one lamination. Take a convergent sequence in Fréchet topology, that consists of geodesics sharing exactly one endpoint with the above geodesic and taking the same positive weight. It is obvious that the extension of the earthquakes to $S^{1}$ corresponding to the sequence does not converge to the extension of the earthquake to $S^{1}$ corresponding to the limit in the quasisymmetric topology. Note that they do converge pointwise. This is in contrast with the statement in Proposition 3.1 which gives the equivalence. However, if we restrict ourselves to the earthquakes on compact surfaces then the equivalence holds. One of the main results in Section 4, 5 is that the equivalence holds for the universal hyperbolic solenoid as well.

\section{Measured laminations on the universal hyperbolic solenoid}

Recall that a leaf of the universal hyperbolic solenoid $\mathscr{Y}$ intersects any local chart countably many times. Each intersection is a 2-disk, which is called a local leaf. Given two local leaves of two global leaves, there exists an identification isometry of the global leaves given as follows. Since the hyperbolic metric in the local charts is continuous for the trivial (vertical) identification, we can choose two points sitting one above the other and two unit tangent vectors based at the points whose 
directions get vertically identified. The two vectors are not necessarily vertically identified because the hyperbolic metrics are not constant in the transverse direction. The isometric identification is uniquely determined by requiring to map one point onto the other such that the unit vector is mapped onto the unit vector. The identification depends on the chart and the choice of two points while the choice of tangent vectors does not affect it.

Fix one local leaf $l$ and consider a sequence of local leaves $l_{n}$ approaching $l$. Suppose we choose two different isometric identifications $f_{n}: \tilde{l} \rightarrow \tilde{l}_{n}$ and $g_{n}: \tilde{l} \rightarrow \tilde{l}_{n}$ of the global leaves $\tilde{l}, \tilde{l}_{n}$ containing local leaves $l, l_{n}$. Note that the identifications differ by the choice of points in the local leaves. Then $g_{n}^{-1} \circ f_{n}$ is an isometry of $\tilde{l}$ which converges to the identity as $n \rightarrow \infty$ because of the continuity in the transverse direction of the hyperbolic metrics. This implies that any two identifications of two global leaves differ by an isometry which is close to the identity when corresponding local leaves are close. Therefore, it makes sense to compare objects preserved by isometries on two nearby leaves as well as maps from leaves.

Definition 4.1. A (transversely continuous) geodesic lamination on the universal hyperbolic solenoid $\mathscr{S}$ is an assignment of a geodesic lamination to each leaf which is continuous, for Hausdorff distance between closed subset of $\mathscr{G}(\boldsymbol{D})$ defined using the angle metric $d$ on $S^{1} \times S^{1}$, with respect to the transverse variations given by each local chart as above.

Namely, we consider the isometric identifications for any local chart as above. The geodesic laminations on global leaves can be mapped to the unit disk $\boldsymbol{D}$ by the identifications. Thus we obtain a map from the local transverse set, obtained by considering each local leaf in the chart as a point, to the space of geodesic laminations on the unit disk $\boldsymbol{D}$. We require that this map be continuous for the Hausdorff topology on the space of geodesic laminations.

This definition certainly seems to be in the spirit of transverse continuity of the hyperbolic metrics on $\mathscr{Y}$. However, we introduce below measured laminations on $\mathscr{S}$ in terms of the continuity of measures. It turns out that the support of measured laminations on $\mathscr{S}$ is not geodesic lamination as above, even though the restriction to each leaf is a geodesic lamination.

Definition 4.2. A (transversely continuous) measured lamination $\mu$ on $\mathscr{Y}$ is an assignment of a bounded measured lamination to each leaf of $\mathscr{S}$ such that it is continuous for the transverse variations with respect to Fréchet topology on the space of measured laminations on the unit disk.

Given a local chart $D \times T$, where $D$ is a 2-disk and $T$ a transverse Cantor set, the measured lamination $\mu$ on $\mathscr{Y}$ gives a map $\mu: T \rightarrow M L_{\mathrm{bdd}}(\boldsymbol{D})$ using the identifications of leaves induced by the local chart. We denote by $M L_{\text {bdd }}(\boldsymbol{D})$ the space of all bounded measured laminations on the unit disk $\boldsymbol{D}$. In the definition 
above, we require that $\left\|\mu(t)-\mu\left(t^{\prime}\right)\right\|_{\nu} \rightarrow 0$ as $t^{\prime} \rightarrow t$ for each $t \in T$ and for each $0<v \leq 1$.

The definition of measured laminations on $\mathscr{Y}$ does not specify the support. To give an example of a measured lamination on $\mathscr{Y}$, fix a measured lamination $\sigma$ on a compact surface $S$ of genus at least two. Since each leaf of $\mathscr{S}$ is a universal cover of the compact surface $S$, we can lift $\sigma$ to a measured lamination $\tilde{\sigma}$ on each leaf of $\mathscr{S}$. The lifts $\tilde{\sigma}$ are locally constant for the transverse variations in the local charts of a TLC hyperbolic metric coming from the hyperbolic metric on $S$. Thus $\tilde{\sigma}$ defines a geodesic lamination on $\mathscr{Y}$.

As we mention above, there are measured laminations on $\mathscr{Y}$ whose supports are not a geodesic laminations on $\mathscr{S}$. We give an example of such a measured lamination.

Example 4.3. We identify $\mathscr{Y}$ with $(\boldsymbol{D} \times \hat{G}) / G$, for a Fuchsian group $G$ uniformizing a compact hyperbolic surface $S=\boldsymbol{D} / G$. We define a measured lamination $\tilde{\mu}$ on $\boldsymbol{D} \times \hat{G}$ which is invariant under $G$. Let $G_{i}$ be a decreasing sequence of finite index normal subgroups of $G=G_{1}$ such that $\cap_{i=1}^{\infty} G_{i}=\{$ id $\}$. We fix two simple closed curves $\gamma_{1}, \gamma_{2}$ in $S$ which intersect in one point and we fix two lifts $\tilde{\gamma}_{1}, \tilde{\gamma}_{2}$ of $\gamma_{1}, \gamma_{2}$ in the universal cover $\boldsymbol{D}$ such that $\left|\tilde{\gamma}_{1} \cap \tilde{\gamma}_{2}\right|=1$. Denote by $C_{1}, C_{2}$ primitive hyperbolic translations in $G$ whose axes are $\tilde{\gamma}_{1}, \tilde{\gamma}_{2}$ respectively. Let $C_{1}^{r_{i}}$ and $C_{2}^{t_{i}}$ be primitive elements in $G_{i}$. We further require that the group $\tilde{G}_{i+1}$ generated by $C_{1}^{r_{i}}, C_{2}^{t_{i}}$ and $G_{i+1}$ is of index at least 3 in $G_{i}$.

Consider the cosets $a_{0}^{i} \tilde{G}_{i+1}, a_{1}^{i} \tilde{G}_{i+1}, \ldots, a_{k_{i}}^{i} \tilde{G}_{i+1}, a_{0}^{i}=\mathrm{id}$, of $\tilde{G}_{i+1}$ in $G$. Since $\left[G_{i}: \tilde{G}_{i+1}\right] \geq 3$, there are at least two cosets different from $\tilde{G}_{i+1}$ which lie in $G_{i}$. Denote them by $a_{1}^{i} \tilde{G}_{i+1}, a_{2}^{i} \tilde{G}_{i+1} \subset G_{i}$. Then $\left(a_{1}^{i} G_{i+1}\right) \cdot\left(a_{2}^{i} G_{i+1}\right)^{-1}$ are not of the form $C_{j}^{k} G_{i+1}$, for $j=1$ or $j=2$ and for some $k \in \mathbb{Z}$. To see this, first note that if $a_{1}^{i}\left(a_{2}^{i}\right)^{-1}$ is a power of $C_{1}$ or $C_{2}$ then it has to be a power of primitive elements $C_{1}^{r_{i}}$ or $C_{2}^{t_{i}}$ in $G_{i}$. Otherwise $\left(a_{1}^{i} G_{i+1}\right) \cdot\left(a_{2}^{i} G_{i+1}\right)^{-1} \notin G_{i} / G_{i+1}$, which is a contradiction. On the other hand, by our choice of $\tilde{G}_{i+1}$ and cosets $a_{1}^{i} \tilde{G}_{i+1}, a_{2}^{i} \tilde{G}_{i+1}$, we get that $a_{1}^{i}\left(a_{2}^{i}\right)^{-1}$ is not a power of primitive elements $C_{1}^{r_{i}}$ or $C_{2}^{t_{i}}$.

Let $\delta_{\tilde{\gamma}_{i}, t}$ denote a unit mass measure on the space of geodesics of $\boldsymbol{D} \times \hat{G}$ supported on the geodesic $\left(\tilde{\gamma}_{i}, t\right) \subset \boldsymbol{D} \times\{t\}$, for $t \in \hat{G}$. We define

$$
\tilde{\mu}^{\prime}:=\sum_{i}\left(\sum_{t \in a_{1}^{i} \hat{G}_{i+1}} m_{i} \delta_{\tilde{\gamma}_{1}, t}+\sum_{t \in a_{2}^{i} \hat{G}_{i+1}} m_{i} \delta_{\tilde{\gamma}_{2}, t}\right)
$$

where $m_{i}>0, m_{i} \rightarrow 0$ as $i \rightarrow \infty$ and $\hat{G}_{i+1}<\hat{G}$ is the profinite completion of $G_{i+1}$. The measured lamination $\tilde{\mu}^{\prime}$ varies continuously in the transverse direction for the Fréchet topology on measured laminations of the unit disk. The continuity is immediate at any $t \in \hat{G}$ because $\tilde{\mu}^{\prime}$ is locally constant. 
We define

$$
\tilde{\mu}:=\sum_{A \in G} A^{*}\left(\tilde{\mu}^{\prime}\right)
$$

Then $\tilde{\mu}$ is invariant under the action of $G$. We first show that the support of $\tilde{\mu}$ on each leaf $\boldsymbol{D} \times\{t\}, t \in \hat{G}$, is a geodesic lamination. Let $\omega$ be a fundamental polygon for the action of $G$ in $\boldsymbol{D}$. Then $\omega \times \hat{G}$ is a fundamental set for the action of $G$ on $\boldsymbol{D} \times \hat{G}$. It is enough to show that the support of $\tilde{\mu}$ has no self-intersections on $\omega \times \hat{G}$. Note that the support of $\tilde{\mu}^{\prime}$ has no self-intersections because the cosets of $\hat{G}$ which contain copies of $\tilde{\gamma}_{1}$ and $\tilde{\gamma}_{2}$ in the support are chosen to be disjoint. The only possibility for the support of $\tilde{\mu}$ to have a self-intersection is the case that a coset $a_{1}^{i} \hat{G}_{i+1}$ is mapped onto the coset $a_{2}^{i} \hat{G}_{i+1}$ by $C_{1}^{k}$ for some $k \in \mathbb{Z}$, and similar for $C_{2}$. This is impossible by our choice of cosets.

We claim that $\tilde{\mu}$ is continuous for the transverse variations in the Fréchet topology on the space of measured laminations of the unit disk $\boldsymbol{D}$. First, we can assume that different lifts of $\gamma_{j}, j=1,2$, in $\boldsymbol{D}$ do not belong to a single box of geodesics $Q$ with $L(Q)=\log 2$ by appropriate choice of the group $G$. If we show continuity in this case, the result follows because the convergence of measured laminations in the Fréchet topology is independent of the hyperbolic metric. We already conclude that $\tilde{\mu}^{\prime}$ is continuous for the transverse variations. By taking the pushforward of $\tilde{\mu}^{\prime}$ by $G$, we add some extra support of $\tilde{\mu}$ intersecting fundamental set $\omega \times \hat{G}$. The extra support is obtained by adding $\left(\tilde{\gamma}_{1}, t\right)$ for $t \in C_{1}^{k} a_{1}^{i} \hat{G}_{i+1}, k \in \mathbb{Z}$ and $i=1,2, \ldots$, and by adding $\left(\tilde{\gamma}_{2}, t\right)$ for $t \in C_{2}^{k} a_{2}^{i} \hat{G}_{i+1}, k \in \mathbb{Z}$ and $i=1,2, \ldots$ It is obvious that the restriction of $\tilde{\mu}$ to the part which intersects $\omega \times \hat{G}$ is continuous for the transverse variations at any $t \in \hat{G}-\{\mathrm{id}\}$, similar to $\tilde{\mu}^{\prime}$. The continuity at $t=\mathrm{id}$ follows because $m_{i} \rightarrow 0$. Our assumption that the orbit of $\tilde{\gamma}_{i}$ does not contain two geodesic which lie in a box $Q$ with $L(Q)=\log 2$ implies the continuity of $\tilde{\mu}$.

The measured lamination $\tilde{\mu}$ on $\boldsymbol{D} \times \hat{G}$ descends to a measured lamination $\mu$ on the universal hyperbolic solenoid $\mathscr{Y}$. The continuity of $\mu$ for the transverse variations follows by the continuity of $\tilde{\mu}$. It is clear that the support of $\mu$ is not a geodesic lamination on $\mathscr{S}$ as in Definition 4.1 because it is not a closed set. Moreover, the closure in $\mathscr{Y}$ of the support of $\mu$ is not a geodesic lamination because, on the baseleaf, it consists of the full preimage of the two intersecting geodesics $\gamma_{1}$ and $\gamma_{2}$ on the closed surface $S$.

\section{Earthquake theorem}

We show that any two points in the Teichmüller space $T(\mathscr{S})$ of the universal hyperbolic solenoid $\mathscr{S}$ are connected by an earthquake. First, we need to recall certain facts from [Šarić 2005] about arbitrary points in $T(\mathscr{S})$. 
A TLC solenoid $\mathscr{Y}$ is homeomorphic to $(\boldsymbol{D} \times \hat{G}) / G$, where $\boldsymbol{D}$ is the unit disk. The space $\boldsymbol{D} \times \hat{G}$ is considered as a universal cover of $\mathscr{Y}$. Denote the covering map by $\pi: D \times \hat{G} \rightarrow \mathscr{Y}$. The action of $G$ is given by

$$
A(z, t):=\left(A(z), t A^{-1}\right)
$$

where $A$ can be considered as an element of $\hat{G}$.

A point in $T(\mathscr{S})$ is given by a (differentiable) quasiconformal map $f: \mathscr{Y} \rightarrow X$, where $X$ is the universal hyperbolic solenoid with an arbitrary hyperbolic metric, not necessarily TLC. We introduced the universal (hyperbolic) cover to $X$ and the covering group as follows [Šarić 2005]. The action by $G$ does not introduce identifications to the set $\{0\} \times \hat{G}$ where $0 \in D$. Consider a local chart $D \times T$ for $X$ which contains $f(\pi(\{0\} \times \hat{G}))$ as a vertical set, where $T \equiv \hat{G}$ and $D$ is a 2-disk with center at 0 . To fix the notation, we assume that $f(\pi(\{0\} \times \hat{G}))$ corresponds to $\{0\} \times T \subset D \times T$ in the local chart. We fix unit tangent vectors at the points $f(\pi(\{0\} \times \hat{G}))$ corresponding to the unit tangent vectors under the chart map at the points $\{0\} \times T$ along the positive axis in the chart $D \times T$.

The universal cover for $X$ is, by the definition, $\boldsymbol{D} \times T$ and the covering map $\pi_{X}: \boldsymbol{D} \times T \rightarrow X$ is given by isometrically mapping each $\boldsymbol{D} \times\{t\}$ onto the leaf containing $f(\pi(0, t))$ such that the origins are mapped onto the origins and the unit tangent vectors along positive axes are mapped onto the unit tangent vectors along the positive axes when considered in the chart $D \times T$. The map $f: \mathscr{Y} \rightarrow X$ lifts to a quasiconformal map

$$
\tilde{f}: \boldsymbol{D} \times \hat{G} \rightarrow \boldsymbol{D} \times T
$$

of the universal covers.

The action of $G$ is conjugated by $\tilde{f}$ to an action of a group $G_{X}$ on $\boldsymbol{D} \times T$. An element $A$ of $G$ acts on $\boldsymbol{D} \times \hat{G}$ by $A(z, t)=\left(A(z), t A^{-1}\right)$, namely it acts on the unit disk component by hyperbolic isometry and it shifts the leaf $\boldsymbol{D} \times\{t\}$ onto the leaf $\boldsymbol{D} \times\left\{t A^{-1}\right\}$. In particular, the action on the disk coordinate is independent of the leaf as the second coordinate. We define a covering transformation $A_{f}$ for $X$ by the formula

$$
\left(A_{f} \circ \tilde{f}\right)(z, t)=(\tilde{f} \circ A)(z, t) .
$$

An equivalent definition for $A_{f}$ is

$$
A_{f}(z, t):=\left(\pi_{X}\right)^{-1}\left(\pi_{X}(z, t), t A^{-1}\right),
$$

where $\pi_{X}(z, t) \in \mathscr{Y}$ and $\pi_{X}\left(\cdot, t A^{-1}\right)$ stands for the inverse of the covering map restricted to $\boldsymbol{D} \times\left\{t A^{-1}\right\}$. Recall that, when restricted to each leaf, the covering map is an isometry for the hyperbolic metric on leaves. Thus $A_{f}$ is an isometry on each leaf of $\boldsymbol{D} \times T$; yet it varies with leaves. The group of covering maps for the 
hyperbolic solenoid $X$ is denoted by

$$
G_{X}:=\tilde{f} G \tilde{f}^{-1} .
$$

If all $A_{f}$ in a finite index subgroup of $G_{X}$ are constant in the transverse direction then $X$ has a TLC hyperbolic metric.

We show that earthquakes are transitive in $T(\mathscr{S})$.

Theorem 5.1. A measured lamination $\mu$ on a solenoid $X$ with an arbitrary hyperbolic metric gives a leafwise earthquake map $E_{\mu}$ of $X$ into another solenoid $Y$ with hyperbolic metric such that there exists a (differentiable) quasiconformal map $f$ : $X \rightarrow Y$ whose extension to the boundary of leaves coincides with the extension of $E_{\mu}$. Any two points in the Teichmüller space $T(\mathscr{S})$ of the universal hyperbolic solenoid $\mathscr{S}$ are connected by a unique earthquake along a measured lamination on $\mathscr{S}$.

Proof. We first show that an earthquake map along a measured lamination $\mu$ on the hyperbolic solenoid $X$ gives another hyperbolic solenoid $Y$. We recall that $\mu$ is an assignment of bounded measured laminations to the leaves of $X$ such that it varies continuously for the transverse variations.

Let $f: \mathscr{Y} \rightarrow X$ be a differentiable quasiconformal map, where $\mathscr{S}=(\boldsymbol{D} \times \hat{G}) / G$. Recall the universal cover $\pi_{X}: D \times T \rightarrow X$ and the lift of $\mu$ to a measured lamination $\tilde{\mu}$, which varies continuously for the transverse variations. In other words, $\tilde{\mu}$ : $T \rightarrow M L_{\mathrm{bdd}}(\boldsymbol{D})$, is continuous for the Fréchet topology on the space $M L_{\mathrm{bdd}}(\boldsymbol{D})$ of bounded measured laminations of $\boldsymbol{D}$ and it satisfies the invariance under the action of $G_{f}$, that is

$$
\left(A_{f}(t)\right)^{*}(\tilde{\mu}(t))=\tilde{\mu}\left(t A^{-1}\right)
$$

for all $A_{f} \in G_{f}$, where $A_{f}(t)$ is the hyperbolic isometry of $\boldsymbol{D}$ obtained by restricting $A_{f}$ to a map from $\boldsymbol{D} \times\{t\}$ onto $\boldsymbol{D} \times\left\{t A^{-1}\right\}$.

We consider a family of earthquakes $E_{\tilde{\mu}(t)}: \boldsymbol{D} \rightarrow \boldsymbol{D}$, for $t \in T$, and we normalize them to fix $1, i$ and -1 on the unit circle $S^{1}=\partial \boldsymbol{D}$. We first show that they induce a family of quasisymmetric maps of $S^{1} \times T \equiv \partial(\boldsymbol{D}) \times T$ onto itself which conjugate $G_{f}$ onto another group of leafwise isometries. Let

$$
h_{t}:=\left.E_{\tilde{\mu}(t)}\right|_{S^{1}},
$$

where $h_{t}: 1, i,-1 \mapsto 1, i,-1$. By the above invariance of $\tilde{\mu}$ under the action of $A_{f}$ and by the fact that an earthquake is determined, up to postcomposition with a hyperbolic isometry, by its measure [Thurston 1986], we get

$$
h_{t A^{-1}} \circ A_{f}(t)=A_{*}(t) \circ h_{t},
$$

where $A_{*}(t)$ is a hyperbolic isometry between $\boldsymbol{D} \times\{t\}$ and $\boldsymbol{D} \times\left\{t A^{-1}\right\}$ defined by the equation. To each $A_{f} \in G_{f}$, we assign such $A_{*}$ which is a hyperbolic isometry 
between leaves. The maps $A_{*}$, for all $A_{f} \in G_{f}$, form a group $G_{*}$ isomorphic to $G_{f}$ under the conjugation by $h$.

We claim that $A_{*}(t)$ is continuous in $t$ for the standard topology on the space of hyperbolic isometries of $\boldsymbol{D}$. To see this, it is enough to show that the images of three fixed point on $S^{1}$ vary continuously in $t$. By the above equation, we get

$$
A_{*}(t)=h_{t A^{-1}} \circ A_{f}(t) \circ h_{t}^{-1} .
$$

Since $\tilde{\mu}(t)$ varies continuously in $t$ and since $h_{t}, h_{t A^{-1}}$ are properly normalized earthquakes, we conclude that $h_{t}, h_{t A^{-1}}$ are continuous in $t$ for the topology of pointwise convergence by [Šarić 2007, Proposition 3.3]. By our assumption, $A_{f}(t)$ is continuous in $t$. Thus $A_{*}(t)$ is continuous in $t$.

We need to show that the quotient $(\boldsymbol{D} \times T) / G_{*}$ is quasiconformally equivalent to $X$, i.e., there exists a homeomorphism $g: X \rightarrow(D \times T) / G_{*}$ which is a differentiable quasiconformal map on each leaf and which varies continuously in the transverse direction in the $C^{\infty}$-topology. Let $g_{t}=\operatorname{ex}\left(h_{t}: \boldsymbol{D} \rightarrow \boldsymbol{D}\right)$ be the barycentric extension of $h_{t}: S^{1} \rightarrow S^{1}$ (see [Douady and Earle 1986] for this definition and for the rest of this paragraph). Since the family $h_{t}, t \in T$, is continuous in the pointwise convergence topology, the family $g_{t}$ of barycentric extensions is continuous for the $C^{\infty}$-topology of $C^{\infty}$-maps over compact subsets of $\boldsymbol{D}$.

We claim that $f_{t}$ is also continuous in the parameter $t$ for the quasiconformal topology. (That the earthquake measures converge in the Fréchet topology does not imply that the extensions of earthquakes to $S^{1}$ converge in the quasisymmetric topology, by the example in Section 3. At this point, we rely heavily on the compactness of the solenoid $X$.) Recall from [Šaric 2004b] that $G_{X}$ has a compact fundamental set for the action on $\boldsymbol{D} \times T$, given by the image under $\tilde{f}$ of the fundamental set $\omega \times \hat{G}$ for the action of $G$ on $\boldsymbol{D} \times \hat{G}$. Thus the Beltrami coefficients of the family $g_{t}$ are continuous in $t$ for the supremum norm over the fundamental set of $X$. By the invariance of the quasisymmetric family $h_{t}$ and by the conformal naturality of barycentric extension [Douady and Earle 1986], we obtain

$$
g_{t A^{-1}} \circ A_{f}(t)=A_{*}(t) \circ g_{t},
$$

for $t \in T$. The invariance under $G_{f}$ and the continuity of Beltrami coefficients of $g_{t}$ on the fundamental domain of $G_{f}$ imply that the Beltrami coefficients of $g_{t}$ are continuous in $t$ for the essential supremum norm on the unit disk. Thus we have obtained a differentiable quasiconformal homeomorphism

$$
\tilde{g}: \boldsymbol{D} \times T \rightarrow \boldsymbol{D} \times T, \quad \tilde{g}(\cdot, t):=g_{t}(\cdot),
$$

which conjugates $G_{f}$ onto $G_{*}$. Consequently, it projects onto a quasiconformal homeomorphism $g: X \rightarrow(D \times T) / G_{*}$. Thus the earthquake $E_{\mu}$ defines a new hyperbolic solenoid $Y:=(\boldsymbol{D} \times T) / G_{*}$ which is the image of $X$. By its definition, 
the boundary values of each $g_{t}$ agree with $\left.E_{\tilde{\mu}(t)}\right|_{S^{1}}$. This finishes the proof of the first part of the Theorem.

It remains to show that any two points $[f: \mathscr{Y} \rightarrow X],[g: \mathscr{S} \rightarrow Y]$ are connected by an earthquake along a measured lamination on $X$. In other words, we need to find a measured lamination $\mu$ on $X$ such that $E_{\mu}$ maps $X$ onto $Y$ and that the extensions of $E_{\mu}$ to the boundaries of leaves are equal to the extensions of $g \circ f^{-1}$. We lift the maps $f$ and $g$ to the maps

$$
\tilde{f}: \boldsymbol{D} \times T \rightarrow \boldsymbol{D} \times T, \quad \text { and } \quad \tilde{g}: \boldsymbol{D} \times T \rightarrow \boldsymbol{D} \times T
$$

of the universal covers of $X$ and $Y$. Let $h_{t}:=\left.\tilde{g} \circ \tilde{f}^{-1}\right|_{\boldsymbol{D} \times\{t\}}$. Note that the family of quasisymmetric maps $h_{t}, t \in T$, is continuous in $t$ for the quasisymmetric topology. By Thurston's earthquake theorem for the unit disk [1986], there exists a measured lamination $\tilde{\mu}(t)$ such that $\left.E_{\tilde{\mu}(t)}\right|_{S^{1}}=h_{t}$. Moreover, $\tilde{\mu}(t)$ is a bounded earthquake measure on $\boldsymbol{D}$. Since $h_{t}$ varies continuously, we get that $\tilde{\mu}(t)$ varies continuously in $t$ for the Fréchet topology by Proposition 3.2. The family $h_{t}$ satisfies invariance properties with respect to $G_{X}$. Therefore, by the uniqueness of earthquake measures [Thurston 1986], the family of corresponding earthquake measures $\tilde{\mu}(t)$ also satisfies invariance properties. Thus it descends to the desired earthquake measure $\mu$ on $X$.

We recall that Proposition 3.2 states that if quasisymmetric maps are close in the quasisymmetric topology then the corresponding earthquake measures are close in the Fréchet topology. The inverse is false in general. However, we show above that the compactness of the universal hyperbolic solenoid $\mathscr{Y}$ forces the continuity of quasisymmetric maps on nearby leaves obtained by earthquaking along transversely continuous measured laminations. The proof extends along the same lines to show that if two measured laminations on the solenoid $\mathscr{Y}$ are close in the Fréchet topology then the extension of earthquake maps to the boundary leaves are close in the quasisymmetric topology. We obtain:

Corollary 5.2. The earthquake map which assigns to each bounded measured lamination on the universal hyperbolic solenoid $\mathscr{S}$ the corresponding marked hyperbolic solenoid is a homeomorphism between the space $M L(\mathscr{S})$ of bounded measured laminations and the Teichmüller space $T(\mathscr{S})$.

\section{Thurston's boundary for $T(\mathscr{Y})$}

We recall the definition of the Liouville map $\mathscr{L}: T(\boldsymbol{D}) \rightarrow H(\boldsymbol{D})$ from the universal Teichmüller space $T(\boldsymbol{D})$ to the space of Hölder distributions $H(\boldsymbol{D})$ of the unit disk $\boldsymbol{D}$. Liouville map first appears in [Bonahon 1988] in the case of the Teichmüller space of a compact Riemann surface and it is used in [Šarić 2005] to introduce Thurston-type boundary to the universal Teichmüller space $T(\boldsymbol{D})$. 
The universal Teichmüller space $T(\boldsymbol{D})$ is the set of all quasisymmetric maps $h: S^{1} \rightarrow S^{1}$ which fix $1, i,-1$. The topology on $T(\boldsymbol{D})$ is defined by requiring that two quasisymmetric maps are close if there exist their quasiconformal extensions to $\boldsymbol{D}$ whose Beltrami coefficients are close in the essential supremum norm on $\boldsymbol{D}$.

The Liouville map $\mathscr{L}: T(\boldsymbol{D}) \rightarrow H(\boldsymbol{D})$ is defined by the pullback

$$
\mathscr{L}(h):=h_{*}(L)
$$

of the Liouville measure $L$ by the quasisymmetric maps $h \in T(\boldsymbol{D})$. The Liouville map $\mathscr{L}$ is a homeomorphism of $T(\boldsymbol{D})$ onto its image; the image $\mathscr{L}(T(\boldsymbol{D})) \subset H(\boldsymbol{D})$ is closed and unbounded [Šarić 2005]. An asymptotic ray to $L(T(\boldsymbol{D}))$ is a path $t \Psi, t>0$ and $\Psi \in H(\boldsymbol{D})$, such that there exists a path $\alpha_{t}, t>0$, in $T(\boldsymbol{D})$ with

$$
t^{-1} \alpha_{t} \rightarrow \Psi
$$

as $t \rightarrow \infty$. Each positive ray through the origin intersects the image $L(T(G))$ in at most one point. Therefore, under the projection of the vector space $H(\boldsymbol{D})$ to the unit sphere in $H(\boldsymbol{D})$, for a fixed $v$-norm, the set $\mathscr{L}(T(\boldsymbol{D}))$ is mapped homeomorphically and its boundary corresponds to the asymptotic rays. Thus we consider asymptotic rays to $\mathscr{L}(T(\boldsymbol{D}))$ as a natural boundary to $T(\boldsymbol{D})$. In [Šarić 2005], we characterize the boundary points of the universal Teichmüller space $T(\boldsymbol{D})$ as all asymptotic rays along bounded measured laminations. Namely,

Theorem 6.1 [Šarić 2005]. The Liouville map $\mathscr{L}: T(\boldsymbol{D}) \rightarrow H(\boldsymbol{D})$ is a homeomorphism onto its image and $\mathscr{L}(T(\boldsymbol{D}))$ projects homeomorphically to the unit sphere. The boundary of $T(\boldsymbol{D})$ is identified by the above embedding with the space of bounded projective measured laminations $P M L_{\mathrm{bdd}}(\boldsymbol{D})$. The quasiconformal mapping class group $Q M C G(\boldsymbol{D})$ acts continuously on the closure $T(\boldsymbol{D}) \cup P M L_{\mathrm{bdd}}(\boldsymbol{D})$ of the universal Teichmüller space $T(\boldsymbol{D})$.

We introduce a Thurston-type boundary to the Teichmüller space $T(\mathscr{Y})$ of the universal hyperbolic solenoid $\mathscr{Y}$. The space of geodesics on $\mathscr{S}=(\boldsymbol{D} \times \hat{G}) / G$ is naturally identified with the $G$-orbits of points in $\left(S^{1} \times S^{1}-\operatorname{diag}\right) \times \hat{G}$ given by lifting a single geodesic on a leaf of $\mathscr{Y}$ to the universal cover $\boldsymbol{D} \times \hat{G}$. Since each leaf of $\mathscr{Y}$ is isometric to the hyperbolic plane, it supports the Liouville measure on the space of its geodesics. Thus $\mathscr{Y}$ has a leafwise Liouville measure which lifts to a leafwise measure, called the leafwise Liouville measure $L_{\text {leaf }}$, on the space of geodesics of the universal cover $\boldsymbol{D} \times \hat{\boldsymbol{G}}$.

A (leafwise) Hölder distribution $\Psi$ for the universal hyperbolic solenoid $\mathscr{Y}$ is a family of Hölder distributions $\Psi_{t} \in H(\boldsymbol{D})$, for $t \in \hat{G}$, which are invariant under the action of $G$, that is

$$
\Psi_{t A^{-1}}\left(\varphi \circ A^{-1}\right)=\Psi_{t}(\varphi)
$$


where $\varphi$ is a Hölder continuous function with compact support on the space of geodesics $G(\boldsymbol{D})$, and which vary continuously in $t$ for the Fréchet topology, that is

$$
\left\|\Psi_{t}-\Psi_{t_{1}}\right\|_{\nu} \rightarrow 0
$$

as $t_{1} \rightarrow t$ for each $t \in \hat{G}$ and for each $0<v \leq 1$.

The $\nu$-norm of a leafwise Hölder distribution $\Psi$ is given by

$$
\|\Psi\|_{\nu}:=\sup \left\{\left|\Psi_{t}(\varphi)\right|: t \in \hat{G}, \varphi \in \operatorname{test}(\nu)\right\},
$$

for $0<v \leq 1$, where test $(v)$ is the set of $v$-test functions on $\mathscr{G}(\boldsymbol{D})$. If $\|\Psi\|_{\nu}<\infty$ for all $0<v \leq 1$ then $\Psi$ is called bounded leafwise Hölder distribution. The space of all bounded leafwise Hölder distributions for the universal hyperbolic solenoid $\mathscr{Y}$ is denoted by $H(\mathscr{S})$.

Let $[f: \mathscr{Y} \rightarrow X] \in T(\mathscr{S})$ be an arbitrary point and denote by $\tilde{f}: \boldsymbol{D} \times \hat{G} \rightarrow \boldsymbol{D} \times T$ the lift of $f$ to the universal cover. Let $h: S^{1} \times \hat{G} \rightarrow S^{1} \times T$ be the leafwise quasisymmetric extension of $\tilde{f}$ to the boundary of leaves. We define the Liouville map $\mathscr{L}_{\mathscr{S}}: T(\mathscr{S}) \rightarrow H(\mathscr{Y})$ for the universal hyperbolic solenoid $\mathscr{Y}$ by

$$
\mathscr{L}_{\mathscr{S}}([f])=h_{*}\left(L_{\text {leaf }}\right) .
$$

Note that bounded measures on $\mathscr{G}(\boldsymbol{D})$ are in $H(\boldsymbol{D})$ and that a pullback by a quasisymmetric of the Liouville measure is bounded [Šarić 2005]. Thus the image of the Liouville map is in $H(\boldsymbol{D})$ and the leafwise statement for the universal hyperbolic solenoid immediately follows.

We show that the Liouville map is an embedding and that the natural boundary, that is the set of asymptotic rays, is homeomorphic to the space of projective measured laminations on $\mathscr{Y}$.

Theorem 6.2. The Liouville map $\mathscr{L}_{\mathscr{S}}: T(\mathscr{Y}) \rightarrow H(\mathscr{Y})$ is a homeomorphisms onto its image. The set of asymptotic rays to $\mathscr{L}_{\mathscr{S}}(T(\mathscr{S}))$ is homeomorphic to the space of projective measured laminations on $\mathscr{S}$. The baseleaf preserving mapping class group $\operatorname{MCG}_{B L P}(\mathscr{Y})$ acts continuously on the closure $T(\mathscr{Y}) \cup P M L(\mathscr{Y})$ of the Teichmüller space $T(\mathscr{S})$ of the universal hyperbolic solenoid $\mathscr{S}$.

Proof. The Liouville map $\mathscr{L}_{\mathscr{S}}$ assigns to any $[f] \in T(\mathscr{S})$ the pullbacks of the leafwise Liouville measures on $\mathscr{G}(\boldsymbol{D} \times \hat{G})$ by the extensions $h$ to $S^{1} \times \hat{G}$ of the lift $\tilde{f}: \boldsymbol{D} \times \hat{G} \rightarrow \boldsymbol{D} \times \hat{G}$. The continuity of $\mathscr{L}: T(\boldsymbol{D}) \rightarrow H(\boldsymbol{D})$ implies that $\left(h_{t}\right)_{*}(L)$ is continuous in $t$ for the Fréchet topology. Thus $\mathscr{L}$ maps $T(\mathscr{S})$ into $H(\mathscr{Y})$. Recall that $T(\mathscr{S})$ embeds in the universal Teichmüller space $T(\boldsymbol{D})$ by restricting the map $f: \mathscr{S} \rightarrow X$ to the baseleaf of $\mathscr{S}$ [Nag and Sullivan 1995]. Denote by $T_{\text {restr }}(\boldsymbol{D})$ the image of the embedding. Also, since the baseleaf is dense in $\mathscr{Y}$, the restriction to the baseleaf of the pullback of the leafwise Liouville measure completely determines the measure. Therefore, the restriction of the Liouville map 
$\mathscr{L}_{\mathscr{S}}$ to the baseleaf, which is equivalent to $\boldsymbol{D}, \mathscr{L}: T_{\text {restr }}(\boldsymbol{D}) \rightarrow H(\boldsymbol{D})$ completely determines the map. Since $\mathscr{L}: T(\boldsymbol{D}) \rightarrow H(\boldsymbol{D})$ is a homeomorphism onto its image, it follows that $\mathscr{L}: T_{\text {restr }}(\boldsymbol{D}) \rightarrow H(\boldsymbol{D})$ is also a homeomorphism onto its image. Therefore, $\mathscr{L}_{\mathscr{S}}: T(\mathscr{S}) \rightarrow H(\mathscr{S})$ is a homeomorphisms onto its image.

Let $s \beta, s>0$, be an asymptotic ray to $\mathscr{L}(T(\mathscr{Y}))$ in $H(\mathscr{Y})$, that is there exists a path $s \mapsto \alpha_{s}$ in $\mathscr{L}(T(\mathscr{S}))$ such that the lifts $\tilde{\alpha}_{s}, \tilde{\beta}$ to the universal cover $\boldsymbol{D} \times \hat{G}$ satisfy $\left\|s^{-1} \tilde{\alpha}_{s}-\tilde{\beta}\right\|_{v} \rightarrow 0$ as $s \rightarrow \infty$, for all $0<v \leq 1$. This implies that

$$
\left\|\left.\tilde{\alpha}_{S}\right|_{\boldsymbol{D} \times\{t\}}-\left.\tilde{\beta}\right|_{\boldsymbol{D} \times\{t\}}\right\|_{\nu} \rightarrow 0
$$

for all $t \in \hat{G}$ as $s \rightarrow \infty$. By [Šrarić 2005], each $\left.\tilde{\beta}\right|_{D \times\{t\}}, t \in \hat{G}$, is a measured lamination on $\boldsymbol{D} \times\{t\}$. Therefore, $\beta \in H(\mathscr{S})$ is a leafwise measured lamination, and since it belongs to $H(\mathscr{Y})$, it is continuous for the transverse variations. Thus $\beta$ is a measured lamination on $\mathscr{Y}$. The earthquake theorem (Theorem 5.1) for the universal hyperbolic solenoid $\mathscr{Y}$ shows that an earthquake path $E_{s \mu}: \mathscr{Y} \rightarrow X_{s}, s>0$, is in $T(\mathscr{Y})$. Denote by $\tilde{\mu}$ the lift of $\mu$ to the universal cover $\boldsymbol{D} \times \hat{G}$ of $\mathscr{Y}$. Then $h_{s}:=E_{s \tilde{\mu}}$ is a leafwise earthquake map. By [Šarić 2005, Theorem 2], we have

$$
\left.s^{-1}\left(\left.h_{S}\right|_{\boldsymbol{D} \times\{t\}}\right)_{*}(L) \rightarrow \tilde{\mu}\right|_{\boldsymbol{D} \times\{t\}},
$$

in the $v$-norm, $0<v \leq 1$, as $s \rightarrow \infty$ for each $t \in \hat{G}$. Moreover, [Šarić 2005, Lemma 4.4] shows that the above convergence in the $v$-norm, $0<v \leq 1$, is uniform, independent of the leaf $\boldsymbol{D} \times\{t\}$. Thus

$$
\left(h_{s}\right)_{*}\left(L_{\text {leaf }}\right) \rightarrow \mu,
$$

as $s \rightarrow \infty$ in the Fréchet topology on $H(\mathscr{Y})$. Accordingly, the natural boundary to $T(\mathscr{S})$, that is the space of asymptotic rays to $\mathscr{L}(T(\mathscr{Y}))$ in $H(\mathscr{Y})$, is homeomorphic to the space $P M L(\mathscr{S})$ of projective measured laminations on $\mathscr{Y}$. The continuity of the action of the baseleaf preserving mapping class group on the closure $T(\mathscr{S}) \cup$ $P M L(\mathscr{S})$ is immediate from [Šarić 2005].

Note that leafwise measured laminations on $\mathscr{Y}$ are given in terms of continuity for the transverse variations. We show that each leafwise measured lamination on $\mathscr{Y}$ is approximated by a transversely locally constant (TLC) measured lamination, that is a lamination obtained by lifting the lamination on compact surfaces to $\mathscr{Y}$, which is parallel to the statement that each hyperbolic metric on $\mathscr{Y}$ is approximated by a TLC hyperbolic metric.

Theorem 6.3. The subset of all measured laminations on the universal hyperbolic solenoid $\mathscr{S}$ that are locally transversely constant $(T L C)$ is dense in the space of all measured laminations $M L(\mathscr{Y})$ on $\mathscr{Y}$ for the Fréchet topology.

Proof. Recall that $T_{\text {restr }}(\boldsymbol{D})$ is obtained by taking the closure of the union of all quasisymmetric maps which conjugate finite index subgroups of $G$ onto other Fuchsian 
groups [Nag and Sullivan 1995], that is the closure of the union

$$
\bigcup_{[G: K]<\infty} \tilde{T}(\boldsymbol{D} / K)
$$

of the lifts $\tilde{T}(\boldsymbol{D} / K)$ to $T(\boldsymbol{D})$ of all Teichmüller spaces $T(\boldsymbol{D} / K)$ of finite degree unbranched covers $\boldsymbol{D} / K$ of $\boldsymbol{D} / G$. By the characterization of the image of $\mathscr{L}$ from [Bonahon 1988], the image $\mathscr{L}\left(\bigcup_{[G: K]<\infty} \tilde{T}(\boldsymbol{D} / K)\right)$ of the union consists of all bounded Hölder distributions $\alpha$ which are positive measures invariant under finite index subgroups of $G$ and which satisfy

$$
e^{-\alpha([a, b] \times[c, d])}+e^{-\alpha([b, c] \times[d, a])}=1
$$

for all $a, b, c, d \in S^{1}$ given in counterclockwise order.

Since $\mathscr{L}$ is a homeomorphism onto its image and $\mathscr{L}(T(\boldsymbol{D}))$ is closed in $H(\boldsymbol{D})$ [Šarić 2005], it follows that $\mathscr{L}\left(T_{\text {restr }}(\boldsymbol{D})\right)$ equals to the closure in the Fréchet topology of $\mathscr{L}\left(\bigcup_{[G: K]<\infty} \tilde{T}(\boldsymbol{D} / K)\right)$. By [Bonahon 1988] or [Šarić 2005], the asymptotic rays to $\mathscr{L}\left(\bigcup_{[G: K]<\infty} \tilde{T}(\boldsymbol{D} / K)\right)$ contain all the projective measured laminations that are invariant under all finite index subgroups $K$ of $G$. The Liouville map $\mathscr{L}$ composed with the projection $p r: H(\mathscr{S}) \rightarrow S_{v}$ to the unit sphere $S_{v}$ in $H(\mathscr{S})$ for a fixed $v$-norm is a homeomorphism [Šarić 2005]. This implies that the points in the closure of $p r \circ \mathscr{L}\left(T_{\text {restr }}(\boldsymbol{D})\right)$ which are not in $p r \circ \mathscr{L}\left(T_{\text {restr }}(\boldsymbol{D})\right)$ are projectivized asymptotic rays to $\mathscr{L}\left(T_{\text {restr }}(\boldsymbol{D})\right)$. Any such point in the closure of $p r \circ \mathscr{L}\left(T_{\text {restr }}(\boldsymbol{D})\right)$ is approximated by a projective measure in $p r \circ \mathscr{L}\left(T_{\text {restr }}(\boldsymbol{D})\right)$ that is invariant under finite index subgroups of $G$. Thus the closure of all invariant, projective measured laminations under finite index subgroups of $G$ contains all asymptotic rays to $\mathscr{L}\left(T_{\text {restr }}\right)$. The measured laminations invariant under finite index subgroups of $G$ lift to locally transversely constant measured laminations on $\mathscr{T}$. Thus the limits of locally transversely constant measured laminations on $\mathscr{Y}$ give all transversely continuous measured laminations on $\mathscr{S}$.

Remark 6.4. The set of asymptotic rays to $\mathscr{L}\left(T_{\text {restr }}(\boldsymbol{D})\right)$ in $H(\boldsymbol{D})$ is equal to the restriction to the baseleaf of the set of asymptotic rays to $\mathscr{L}(T(\mathscr{S}))$ in $H(\mathscr{S})$. This is a consequence of the proof of Theorem 6.3, since each asymptotic ray for $\mathscr{L}\left(T_{\text {restr }}(\boldsymbol{D})\right)$ is the limit in the Fréchet topology of the asymptotic rays invariant under finite index subgroups of $G$.

\section{The punctured solenoid}

We sketch an extension of our results to the punctured solenoid $\mathscr{Y}_{p}$ defined in [Penner and Šarić 2005]. We first recall the definition of $\mathscr{Y}_{p}$. 
Let $H$ be the subgroup of $P S L_{2}(\mathbb{Z})$ such that $\boldsymbol{D} / H$ is a once punctured torus. Denote by $\hat{H}$ the profinite completion of $H$. We define the punctured solenoid by

$$
\mathscr{S}_{p}:=(\boldsymbol{D} \times \hat{H}) / H,
$$

where the action of $H$ is given by $A(z, t):=\left(A(z), t A^{-1}\right)$, for $A \in H$.

A leafwise measured lamination on $\mathscr{Y}_{p}$ is an assignment of a bounded measured lamination to each leaf of $\mathscr{S}_{p}$ which varies continuously in the transverse direction. The support of a leafwise measured lamination on $\mathscr{S}_{p}$ is a leafwise geodesic lamination which is not necessarily continuous for the transverse variations. We say that the support of a leafwise measured lamination on $\mathscr{S}_{p}$ is compact if, when restricted to each leaf of $\mathscr{S}_{p}$, the support geodesic lamination is a precompact subset of $\mathscr{Y}_{p}$. The earthquake theorem (Theorem 5.1) holds for $\mathscr{S}_{p}$ when we use the space of measured laminations with compact support $M L_{0}\left(\mathscr{S}_{p}\right)$.

If a simple geodesic on a punctured surface enters a definite neighborhood of a puncture, then it has an endpoint at the puncture. We recall a standard proof of this fact in the upper halfplane model of the hyperbolic plane. Let $A: z \mapsto z+1$ be the parabolic element corresponding to the puncture. If a lift of the geodesic entering a neighborhood of a puncture on the surface is a Euclidean halfcircle with radius greater than $1 / 2$, then the translate of the lift of the geodesic by $A$ intersects the lift of the geodesic. Thus the geodesic is not simple. Contradiction. Therefore, there exists a definite neighborhood of a puncture where no simple geodesic enters unless it ends at the puncture.

Assume that a measured lamination $\mu$ on $\mathscr{S}_{p}$ does not have a compact support. Then there exists a leaf $l$ of $\mathscr{S}_{p}$ such that the restriction $\mu_{l}$ of $\mu$ contains a geodesic $g \in l$ with an endpoint at the puncture. By the continuity for the transverse variations, the support of $\mu_{l}$ contains the translates of $g$ in $l$ by parabolic elements $A^{k n} \in H$, for a fixed $k>0$ and for all $n \in \mathbb{N}$, where $A$ fixes the endpoint of $g$. Then $g$ has to be isolated in $l$ because otherwise the translates under $A^{k n}$ of the geodesics in the support of $\mu_{l}$ converging to $g$ would intersect the geodesics converging to $g$ similar to the punctured surface case. Thus $\mu_{l}$ would not be a measured lamination. The remaining possibility is that $g$ is isolated with atomic measure. Then the translates $A^{k n}(g)$ also have atomic measure approximately equal to the atomic measure of $g$ for $n$ large and they share the same endpoint. Then the measured lamination $\left.\mu\right|_{l}$ is not bounded, which is a contradiction with the definition of a measured lamination on $\mathscr{S}_{p}$. Therefore, a measured lamination $\mu$ on $\mathscr{S}_{p}$ always has a compact support.

As in the proof of Theorem 5.1, it follows that the extension to the boundary of each quasiconformal map $f: \mathscr{Y}_{p} \rightarrow X_{p}$ can be achieved by leafwise earthquakes whose measures vary continuously in the transverse direction for the Fréchet topology on earthquake measures. On the other hand, a measured lamination $\mu$ on $\mathscr{Y}_{p}$ 
with compact support gives a quasiconformal map $f: \mathscr{S}_{p} \rightarrow X_{p}$ whose extensions to the boundaries of the leaves agree with the earthquake $E_{\mu}$. Thus the earthquake theorem (Theorem 5.1) is true for the punctured solenoid on $M L_{0}\left(\mathscr{Y}_{p}\right)$, the space of measured laminations with the compact support on $\mathscr{S}_{p}$.

We explain how to extend Thurston's boundary to the Teichmüller space of the punctured solenoid $\mathscr{S}_{p}$. We show that the boundary consists of $P M L_{0}\left(\mathscr{S}_{p}\right)$. By the extension of the earthquake theorem (Theorem 5.1) and by the proof of Theorem 6.2 , it is only necessary to show that the asymptotic rays to $\mathscr{L}\left(T\left(\mathscr{S}_{p}\right)\right) \subset H\left(\mathscr{S}_{p}\right)$ are of the form $t W, t>0$, for some $W \in M L_{0}\left(\mathscr{Y}_{p}\right)$. Let $\alpha_{t} \in \mathscr{L}\left(T\left(\mathscr{S}_{p}\right)\right)$ be such that $t^{-1} \alpha_{t} \rightarrow W$ as $t \rightarrow \infty$. Then the restriction of $W$ to each leaf of $\mathscr{Y}_{p}$ is a bounded measured lamination by the results in [Šarić 2005]. Since $W$ is continuous for the transverse variations, it follows that $W$ is a measured lamination on $\mathscr{S}_{p}$. By the above, $W \in M L_{0}\left(\mathscr{S}_{p}\right)$.

\section{References}

[Biswas et al. 1999] I. Biswas, M. Mitra, and S. Nag, "Thurston boundary of Teichmüller spaces and the commensurability modular group", Conform. Geom. Dyn. 3 (1999), 50-66. MR 2000b:32030 Zbl 0922.32012

[Bonahon 1988] F. Bonahon, "The geometry of Teichmüller space via geodesic currents", Invent. Math. 92:1 (1988), 139-162. MR 90a:32025 Zbl 0653.32022

[Bonnot et al. 2006] S. Bonnot, R. Penner, and D. Šarić, "A presentation for the baseleaf preserving mapping class group of the punctured solenoid", preprint, 2006. math/0608066

[Candel 1993] A. Candel, "Uniformization of surface laminations", Ann. Sci. École Norm. Sup. (4) 26:4 (1993), 489-516. MR 94f:57025 Zbl 0785.57009

[Douady and Earle 1986] A. Douady and C. J. Earle, "Conformally natural extension of homeomorphisms of the circle”, Acta Math. 157:1-2 (1986), 23-48. MR 87j:30041 Zbl 0615.30005

[Epstein et al. 2006] A. Epstein, V. Markovic, and D. Šarić, "Extremal maps for the universal hyperbolic solenoid", preprint, 2006, Available at ftp://ftp.math.sunysb.edu/preprints/ims06-02.pdf.

[Fathi et al. 1979] A. Fathi, F. Laudenbach, and V. Poenaru, Travaux de Thurston sur les surfaces, Astérisque 66, Société Mathématique de France, Paris, 1979. Séminaire Orsay, with an English summary. MR 82m:57003 Zbl 1024.01017

[Kerckhoff 1983] S. P. Kerckhoff, "The Nielsen realization problem", Ann. of Math. (2) 117:2 (1983), 235-265. MR 85e:32029 Zbl 0528.57008

[Lakic 1997] N. Lakic, “Strebel points”, pp. 417-431 in Lipa's legacy (New York, 1995), edited by J. Dodziuk and L. Keen, Contemp. Math. 211, Amer. Math. Soc., Providence, 1997. MR 99c:32027 Zbl 0957.30014

[Markovic and Šarić 2006] V. Markovic and D. Šarić, "Teichmüller mapping class group of the universal hyperbolic solenoid”, Trans. Amer. Math. Soc. 358:6 (2006), 2637-2650. MR 2006j:30084 Zbl 1095.30040

[Nag and Sullivan 1995] S. Nag and D. Sullivan, "Teichmüller theory and the universal period mapping via quantum calculus and the $H^{1 / 2}$ space on the circle", Osaka J. Math. 32:1 (1995), 1-34. MR 96c:32023 Zbl 0820.30027 
[Odden 1997] C. Odden, Virtual automorphism group of the fundamental group of a closed surface, Ph.D. thesis, Duke University, Durham, 1997.

[Odden 2005] C. Odden, "The baseleaf preserving mapping class group of the universal hyperbolic solenoid”, Trans. Amer. Math. Soc. 357:5 (2005), 1829-1858. MR 2005i:57018 Zbl 1077.57017

[Penner and Šarić 2005] R. C. Penner and D. Šarić, "Teichmüller theory of the punctured solenoid", preprint, 2005, Available at ftp://ftp.math.sunysb.edu/preprints/ims05-06.pdf.

[Šarić 2004a] D. Šarić, "Infinitesimal Liouville distributions for Teichmüller space", Proc. London Math. Soc. (3) 88:2 (2004), 436-454. MR 2004j:30094 Zbl 1051.30041

[Šarić 2004b] D. Šarić, "On quasiconformal deformations of the universal hyperbolic solenoid", preprint, 2004, Available at http://www.math.sunysb.edu/ saric/research/solenoid2.pdf. To appear in J. Anal. Math.

[Šarić 2005] D. Šarić, “Geodesic currents and Teichmüller space”, Topology 44:1 (2005), 99-130. MR 2006g:32019 Zbl 1082.30036

[Šarić 2006] D. Šarić, "Real and complex earthquakes", Trans. Amer. Math. Soc. 358:1 (2006), 233249. MR 2006i:30063 Zbl 02212273

[Šarić 2007] D. Šarić, “Bounded earthquakes”, Proc. Amer. Math. Soc. (2007). To appear.

[Sullivan 1993] D. Sullivan, "Linking the universalities of Milnor-Thurston, Feigenbaum and Ahlfors-Bers", pp. 543-564 in Topological methods in modern mathematics (Stony Brook, NY, 1991), Publish or Perish, Houston, TX, 1993. MR 94c:58060 Zbl 0803.58018

[Thurston 1986] W. P. Thurston, "Earthquakes in two-dimensional hyperbolic geometry", pp. 91112 in Low-dimensional topology and Kleinian groups (Coventry and Durham, 1984), edited by D. B. A. Epstein, London Math. Soc. Lecture Note Ser. 112, Cambridge Univ. Press, Cambridge, 1986. MR 88m:57015 Zbl 0628.57009

[Thurston 1988] W. P. Thurston, "On the geometry and dynamics of diffeomorphisms of surfaces", Bull. Amer. Math. Soc. (N.S.) 19:2 (1988), 417-431. MR 89k:57023 Zbl 0674.57008

Received October 17, 2006.

DRAGOMIR ŠARIĆ

INSTITUTE FOR MATHEMATICAL SCIENCES

STONY BROOK UNIVERSITY

STONY BROOK, NY 11794

UNITED STATES

and

DePartment of Mathematics

QUEENS COLLEGE

City UNIVERSITY OF NEW YorK

65-30 KISSENA BLVD.

FLUSHING, NY 11367

UNITED STATES

saric@math.sunysb.edu 